\title{
Terahertz Nonlinear Spectroscopy of Free-carriers in Direct Bandgap Semiconductors
}

\author{
L. Razzari ${ }^{* a, b}$, F. Blanchard ${ }^{\text {a }}$, F. H. Su ${ }^{\text {c }}$, G. Sharma ${ }^{\mathrm{a}}$, A. Ayesheshim ${ }^{\mathrm{c}}$, T. L. Cocker ${ }^{\mathrm{c}}$, L. V. Titova ${ }^{\mathrm{c}}$, \\ H-C Bandulet ${ }^{\mathrm{a}}$, R. Morandotti ${ }^{\mathrm{a}}, \mathrm{J}_{-} \mathrm{C}$ Kieffer $^{\mathrm{a}}$, T. Ozaki ${ }^{\mathrm{a}}$, M. Reid ${ }^{\mathrm{d}}$, and F. A. Hegmann ${ }^{\mathrm{c}}$ \\ ${ }^{a}$ INRS-EMT, Advanced Laser Light Source, Université du Québec, Varennes, QC, J3X 1S2, Canada \\ ${ }^{b}$ Dipartimento di Elettronica, Università di Pavia, via Ferrata 1, 27100 Pavia, Italy \\ ${ }^{c}$ Department of Physics, University of Alberta, Edmonton, Alberta T6G 2G7, Canada \\ ${ }^{\mathrm{d}}$ Department of Physics, University of Northern British Columbia, Prince George, British Columbia, \\ V2N 4Z9, Canada
}

\begin{abstract}
Nonlinear dynamics of free-carriers in direct bandgap semiconductors at terahertz $(\mathrm{THz})$ frequencies is studied using intense few-cycle pulses. Techniques as Z-scan, THz-pump / THz-probe, and optical-pump/ THz-probe are employed to explore nonlinear interactions in both n-doped and photoexcited systems. The physical mechanism that gives rise to such interactions is found to be intervalley scattering.
\end{abstract}

Keywords: Terahertz pulses, time-resolved terahertz spectroscopy, ultrafast nonlinear optics, semiconductors, z-scan, intervalley scattering

\section{INTRODUCTION}

Ultrafast nonlinear processes have been extensively explored in the visible and near infrared frequency range, thanks to the availability of ultrashort pulses delivered by mode-locked lasers. Here, the combination of high excitation intensities together with a very fine temporal resolution have shed new light on diverse aspects of condensed-matter dynamics [1]. On the other hand, this kind of phenomena has remained relatively unexplored in the $\mathrm{THz}$ spectral region (typically 0.1 $10 \mathrm{THz}$ ), mainly because of the lack of sources delivering high-energy, few-cycle THz pulses. Nowadays, this kind of sources is becoming available [2,3], thus opening the route towards the understanding of new aspects of radiation-matter interaction.

Nonlinear interactions at $\mathrm{THz}$ frequencies possess interesting properties and peculiarities: on one side, in this spectral range one can observe an intermediate regime in which both electronic and ionic motions contribute to the nonlinear dielectric function of a material. On the other side, the very low energy per photon associated to this radiation allows to neglect multiphoton interactions in semiconductors, thus opening up the possibility of observing drift-velocity-based nonlinearities owing to free carriers in this type of systems.

While these processes were studied in the past using THz pulses with a duration of several tens of nanoseconds $[4,5]$, the new-generation few-cycle $\mathrm{THz}$ sources allow now to explore their ultrafast nature and dynamics in the picosecond domain [6].

Our investigation has been aimed at exploring the ultrafast nonlinear dynamics of free carriers in semiconductors and at developing the proper tools for this new kind of THz spectroscopy. The paper is organized as follows. In Section 2, we will describe the large aperture zinc telluride $(\mathrm{ZnTe}) \mathrm{THz}$ source, which has been used to perform the nonlinear $\mathrm{THz}$ experiments described here. In Section 3, we will discuss in detail the experimental observations of the absorption bleaching in $n$-doped InGaAs films revealed by our Z-scan technique. The same effect will be explored further by means of THz-pump - THz-probe spectroscopy in Section 4. Finally, in Section 5 we will report the results of our experiments on photoexcited GaAs using the optical-pump/ THz-probe technique.

*luca.razzari@emt.inrs.ca; phone 1450 929-8202; fax 1450 929-8102; http://www.uop.ca/research-associates/luca-razzari

Copyright 2010 Society of Photo-Optical Instrumentation Engineers. One print or electronic copy may be made for personal use only. Systematic reproduction and distribution, duplication of any material in this paper for a fee or for commercial purposes, or modification of the content of the paper are prohibited. http://dx.doi.org/10.1117/12.858332

Terahertz Emitters, Receivers, and Applications, edited by Jean-François Lampin, Didier J. Decoster, Manijeh Razeghi, Proc. of SPIE Vol. 7763, 77630B · C 2010 SPIE · CCC code: 0277-786X/10/\$18 · doi: 10.1117/12.858332 


\section{TERAHERTZ SOURCE}

In our experiments, high-energy $\mathrm{THz}$ pulses are generated by optical rectification in a large aperture (75 $\mathrm{mm}$ diameter) ZnTe single crystal wafer [2]. The Ti:sapphire laser beam lines of the Advanced Laser Light Source (ALLS) used in these experiments operates at a repetition rate of $100 \mathrm{~Hz}$ and provides $800 \mathrm{~nm}, \sim 40 \mathrm{fs}$ laser pulses with energies as high as $70 \mathrm{~mJ}$ per pulse.

The experimental setup, shown in Fig. 1, comprises three main parts: (i) a THz generation chamber held under vacuum ( $<10-6$ torr), (ii) an 800-nm probe line in air, and (iii) a dry-nitrogen-purged $\mathrm{THz}$ propagation line. The $\mathrm{THz}$ emitter consists of a 1-mm-thick (110) ZnTe single crystal wafer with a diameter of $75 \mathrm{~mm}$. To minimize saturation and to avoid damage to the crystal surface, the $800 \mathrm{~nm}$ beam is spatially expanded to about $36 \mathrm{~cm}^{2}$ at the surface of the ZnTe emitter. Any remaining $800 \mathrm{~nm}$ light transmitted through the ZnTe wafer is blocked using a black polyethylene absorber, which is transparent to $\mathrm{THz}$ radiation.

Five off-axis parabolic mirrors are used after the $\mathrm{THz}$ generating crystal to redirect the $\mathrm{THz}$ wave to the detector, with 3 focus positions: (i) at the exit of the vacuum chamber, (ii) at the sample position for spectroscopy, and (iii) at the ZnTe detector crystal for EO sampling. A chopper positioned at the first focus allows modulation of the $\mathrm{THz}$ beam for lock-in detection. Two 4" diameter wire-grid polarizers (Microtech Instruments model G30L) are used to control the intensity and the polarization of the $\mathrm{THz}$ beam.

To detect the THz pulse waveform, we use free-space electro-optic (EO) sampling in a second, $0.5 \mathrm{~mm}$ thick, (110) ZnTe crystal [7]. A lock-in amplifier connected to the output of a pair of photodiodes and referenced to the chopper is used to acquire the $\mathrm{THz}$ waveforms.

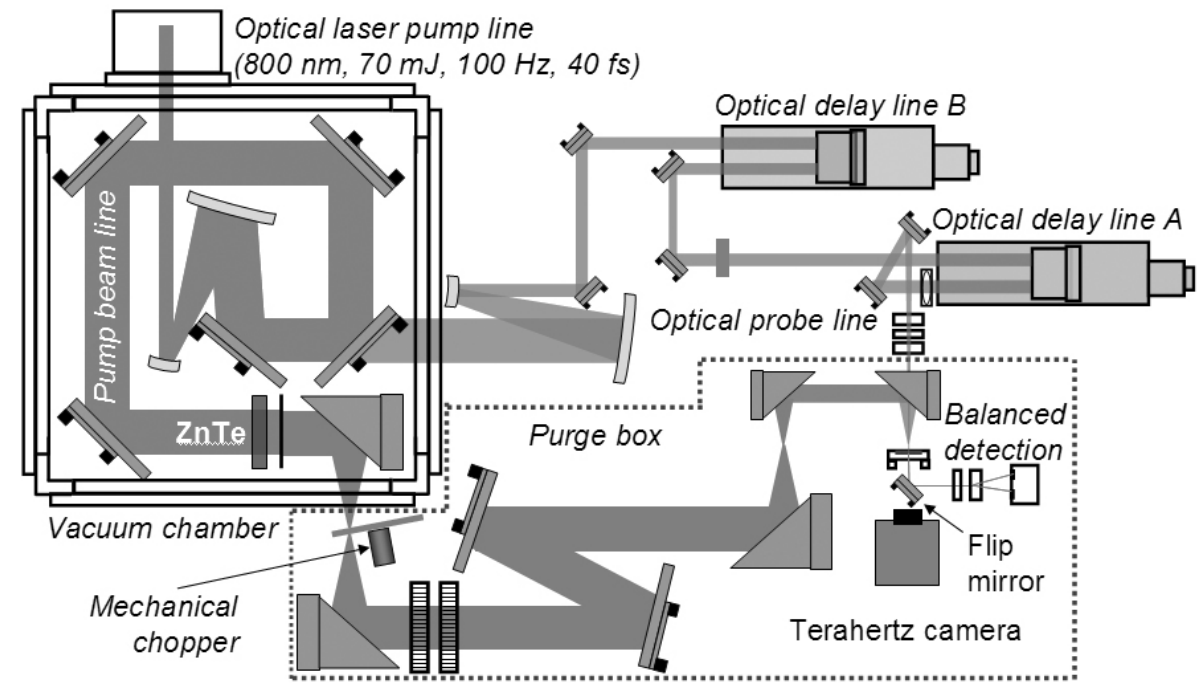

Figure 1. Schematic of the high-intensity THz pulse source at the Advanced Laser Light Source (ALLS).

The source provides picosecond $\mathrm{THz}$ pulses in the frequency range of $0.1-3 \mathrm{THz}$ with $\mu \mathrm{J}$-level energies. These pulses are focused by an off-axis parabolic mirror down to a spot size of $1.6 \mathrm{~mm}$ (full-width at $1 / \mathrm{e}^{2}$ of the maximum) at the second focal position (i.e. the sample position). We used a $\mathrm{BaSrTiO}_{3}$ (BST) pyroelectric infrared camera (Electrophysics model PV320-L2V) to image the THz spot at the focus. This camera operates with an internal $10 \mathrm{~Hz}$ chopper and has a $320 \times 240$ pixel imaging array with a pixel spacing of $48.5 \mu \mathrm{m}$. The $\mathrm{THz}$ beam profile is found to be well fitted by a Gaussian shape. For measuring the THz energy, we used a calibrated pyroelectric energy detector (Coherent Molectron J4-05).

\section{TERAHERTZ Z-SCAN IN N-DOPED INDIUM GALLIUM ARSENIDE}

Using the experimental set-up presented in Section 2, we performed an open aperture Z-scan measurement [8]. Z-scan is a very common and straightforward nonlinear characterization technique used to determine intensity dependent transmission change in a given sample. When performing a Z-scan measurement, the transmittance of the sample is measured as the sample is moved along the propagation path $\mathrm{z}$ of a focused $\mathrm{THz}$ beam. Whenever nonlinear absorption 
takes place, the intensity of the transmitted $\mathrm{THz}$ beam depends on the spot size of the beam at the sample position. Clearly, THz intensity is maximum at the focus and is progressively reduced as the sample is moved away from the focus (in both directions). This method is widely used in multiphoton absorption studies $[9,10]$, and has proven to be effective even for the characterization of saturable absorbers.

The sample we used for this experiment was a thin film of a heavily doped direct bandgap semiconductor, namely Indium Gallium Arsenide. Specifically, it consists of a 500nm thick n-type $\operatorname{In}_{0.53} \mathrm{Ga}_{0.47} \mathrm{As}$ epilayer with a doping concentration of about $2 \times 10^{18} \mathrm{~cm}^{-3}$ grown by metal-oxide chemical vapor deposition on a lattice-matched, $0.5 \mathrm{~mm}$ thick semi-insulating InP substrate. At low excitation levels, the sample transmits about $3 \%$ of the incident $\mathrm{THz}$ energy. We have confirmed that this strong $\mathrm{THz}$ absorption is mainly due to the high conductivity of the epilayer, since measurements of the InP substrate alone have shown an overall $\mathrm{THz}$ transmission (including absorption and reflection losses) of about $60 \%$.

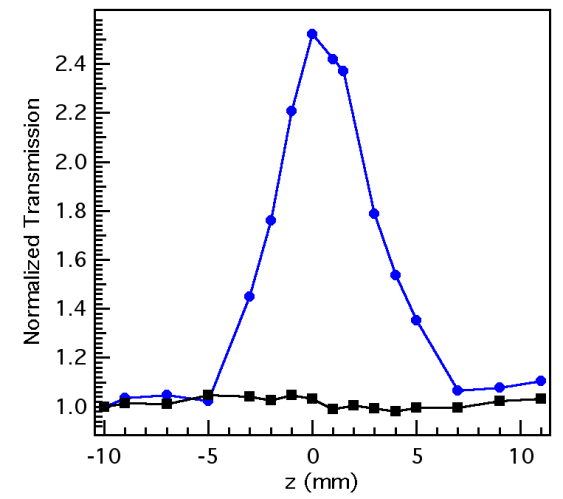

Figure 2. Energy Z-scan traces of the InGaAs epilayer on an InP substrate (blue curve), and of the substrate alone (black curve).

Fig. 2 shows how the energy transmission through the sample varies as a function of the $\mathrm{z}$ position along the scan, with an incident THz pulse energy of $0.8 \mu \mathrm{J}$ (peak THz field $\sim 200 \mathrm{kV} / \mathrm{cm}$ ). A clear bleaching of the absorption is evidenced at the focus of the terahertz beam. The same scan performed on the substrate alone (black curve in Fig. 2) does not return any evidence of the bleaching.

This remarkable phenomenon is attributed to terahertz-electric-field-driven scattering of electrons into satellite valleys of the conduction band. In these satellite valleys, electrons acquire a significantly higher effective mass, reducing the conductivity of the sample and thus increasing the terahertz transmission.
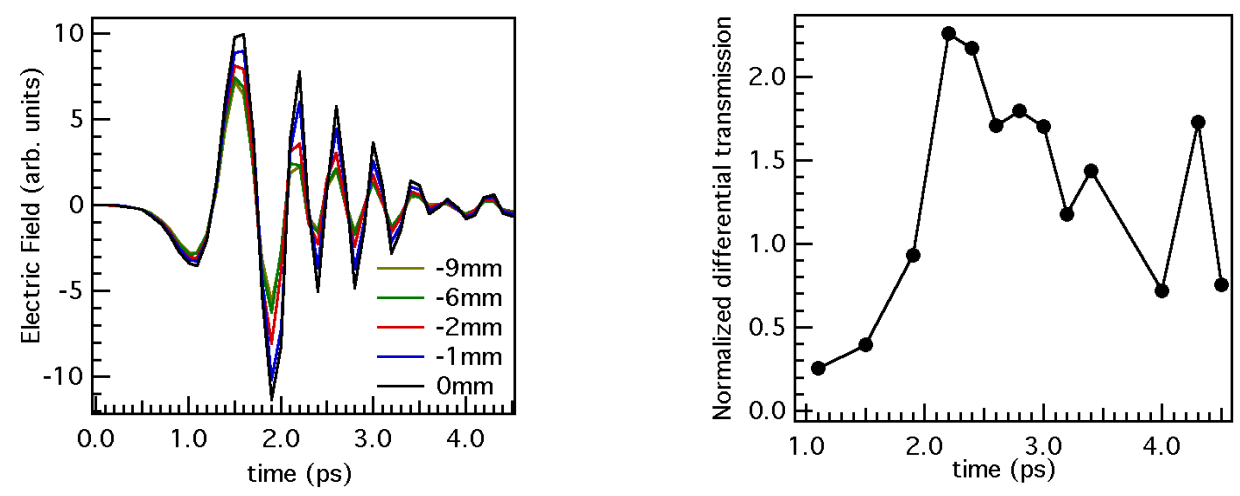

Figure 3. (a) Transmitted electric field for different positions of the Z-scan; (b) Normalized electric field differential transmission as a function of time (the difference is taken between the focal position and a position far away from the focus).

By performing coherent detection of transmitted terahertz pulses, we can improve our understanding of the process, looking into the dynamics of the bleaching mechanism. Fig. 3a shows the transmitted $\mathrm{THz}$ electric field for different positions along the Z-scan. As one can observe, no time shift is present between the traces, clearly showing that changes in the imaginary part of the conductivity are negligible in this specific context. On the other hand, the transmission 
enhancement is found to be not uniform in time, suggesting that the physical mechanism responsible of the bleaching has dynamical features on a time scale comparable with the THz pulse duration.

The dynamics of the bleaching process becomes more evident when the difference in electric field transmission between the focal position of the Z-scan and a position far away from the focus for each peak of the terahertz pulse is plotted, as in Fig. 3b. An initial increase in transmission (with a peak at $t=2.2 \mathrm{ps}$ in Fig. $3 b$ ) is followed by a slower decay over approximately a 2 ps time scale. This behaviour can be explained as follows. Free carriers in the $\Gamma$ valley are accelerated by the terahertz electric field during its oscillations. When the carriers acquire enough kinetic energy to overcome the nearest intervalley separation, they may scatter into an upper valley (i.e. the L-valley) where the effective mass is higher and thus the $\mathrm{THz}$ transmission is enhanced. The electrons in this upper valley will then have a finite probability of scattering back to the $\Gamma$ valley, resulting in a drop in the $\mathrm{THz}$ transmission with a time constant given by the $\mathrm{L} \rightarrow \Gamma$ intervalley relaxation time. It is worth noting that this relaxation time is known to be about $3.1 \mathrm{ps}$ in InGaAs [11], which is consistent with the decay dynamics observed in Fig. 3b. Recent simulations based on a standard Drude model, used to describe the collective motion of the electrons in the conduction band, and incorporating intervalley scattering, have been found to be in good agreement with our experimental findings [12].

\section{THZ-PUMP/THZ-PROBE SPECTROSCOPY}

To confirm the results presented in Section 3, we have set up a more advanced THz-pump / THz-probe characterization experiment. The experimental setup in this case includes an additional $\mathrm{THz}$ probe beam, which is generated by a $10 \times 10 \times 0.5 \mathrm{~mm} \mathrm{ZnTe}$ crystal placed after the gold off-axis parabolic mirror prior to the sample position (second $\mathrm{THz}$ focus in Fig. 1). This THz probe beam was superimposed on to the THz pump beam at its focus. An additional black polyethylene sheet, which is transparent to the THz radiation, was placed before the sample to block any remaining 800$\mathrm{nm}$ light transmitted through the $\mathrm{ZnTe}$ crystal used to generate the THz probe.

In order to avoid crosstalk between the two THz beams, a chopper was inserted in the THz probe line, and the two beams transmitted through the sample were geometrically separated. In addition, we placed a metallic mask to block the remaining $\mathrm{THz}$ pump beam after the sample position.

Fig. 4 shows the normalized transmission of the main peak of the THz probe pulse as a function of the pump-probe delay time. The two curves show the transmission change for a probing polarization perpendicular to the pump beam and the same measurement performed for the parallel configuration, when both beams have the same polarization.

In our case, the closest upper valley of the $\operatorname{In}_{0.53} \mathrm{Ga}_{0.47} \mathrm{As}$ is the $\mathrm{L}$ valley, and the effective masses at the bottom of the two valleys are $\mathrm{m}_{\Gamma}=0.03745 \mathrm{~m}_{\mathrm{e}}$ and $\mathrm{m}_{\mathrm{L}}=0.26 \mathrm{~m}_{\mathrm{e}}$ respectively. By performing the experiment with crossed polarizations, a significant enhancement in the transmission is observed, which follows the population transfer between the $\Gamma$ and $\mathrm{L}$ valley and back (black curve in Fig. 4). The dynamics of this process is in excellent agreement with our previous Z-scan results and can be attributed once again to electric-field-driven intervalley scattering.

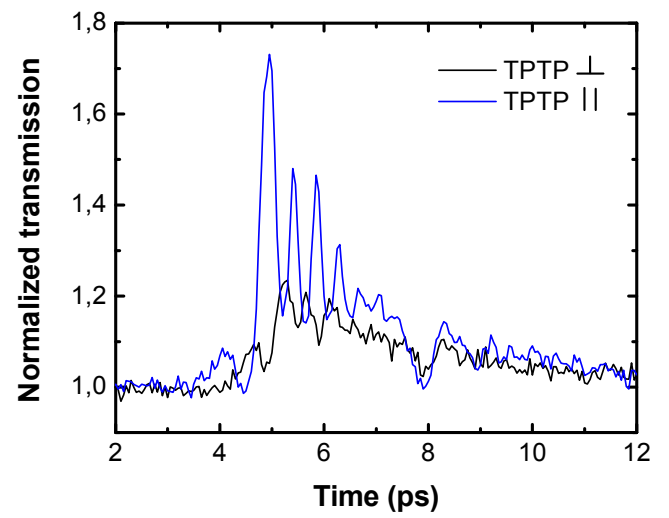

Figure 4. Normalized transmission of the peak THz probe electric field for horizontal THz pump polarization horizontal THz probe polarization (parallel TPTP, $\|$ ) and normalized transmission of the peak THz probe electric field for horizontal THz pump polarization - vertical THz probe polarization (perpendicular TPTP, $\perp$ ).

If one performs instead the same experiment using parallel polarizations (blue curve in Fig. 4), the transmission enhancement is more evident and fast oscillations appear, which seem to follow the shape of the THz pump waveform. 
To understand the differences seen in the two probing cases, we derived the effective masses for perpendicular and parallel conditions [13], using the definition of the energy band that includes the nonparabolicity factor $\alpha$ [14]. The second order derivation turns out to show a direct difference in the effective masses if one probes the electrons for a position far up in the valley, in the parallel or perpendicular direction. This unprecedented result could open up a new way of characterizing the conduction band structure of semiconductors.

\section{OPTICAL-PUMP / TERAHERTZ-PROBE SPECTROSCOPY OF UNDOPED GALLIUM ARSENIDE}

We have also used an optical-pump / intense THz-probe (OPTP) technique to explore the nonlinear electron dynamics of undoped GaAs [15]. The OPTP experiment is performed on a $0.5 \mathrm{~mm}$ thick semi-insulating (SI)-GaAs wafer. Again, we use the intense THz source described in Section 2 for this experiment. The sample is placed at the focus of the $\mathrm{THz}$ beam, and an optical pump beam ( $800 \mathrm{~nm}$ wavelength, $40 \mathrm{fs}$ pulse duration, $8 \mathrm{~mJ} / \mathrm{cm}^{2}$ fluence) is used to photoexcite the sample.

At the sample position, the spot size of the $\mathrm{THz}$ probe beam and the optical pump beam are $1.6 \mathrm{~mm}$ and $12 \mathrm{~mm}$, respectively. Before detection, in the arm where the THz beam is collimated, we placed two wire grid polarizers to keep the $\mathrm{THz}$ electric field within the linear detection regime of the detector crystal. We further used other wire-grid polarizers and $\mathrm{Si}$ wafers to adjust the amplitude of the $\mathrm{THz}$ probe pulse at the sample position. All experiments were performed under dry nitrogen purge at room temperature.

First, we measured the THz transmission, sitting on the peak of the THz pulse, as a function of the pump-probe delay time. This is a common method for probing ultrafast carrier dynamics in semiconductors in linear OPTP [16]. We have studied high and low THz-probe field transmission in photoexcited GaAs, where high and low field correspond to 173 $\mathrm{kV} / \mathrm{cm}$ and $4 \mathrm{kV} / \mathrm{cm}$, respectively. Fig. 5 shows the $\mathrm{THz}$ transmission as a function of pump-probe delay time for high and low THz fields, respectively. The inset shows the long-term dynamics.

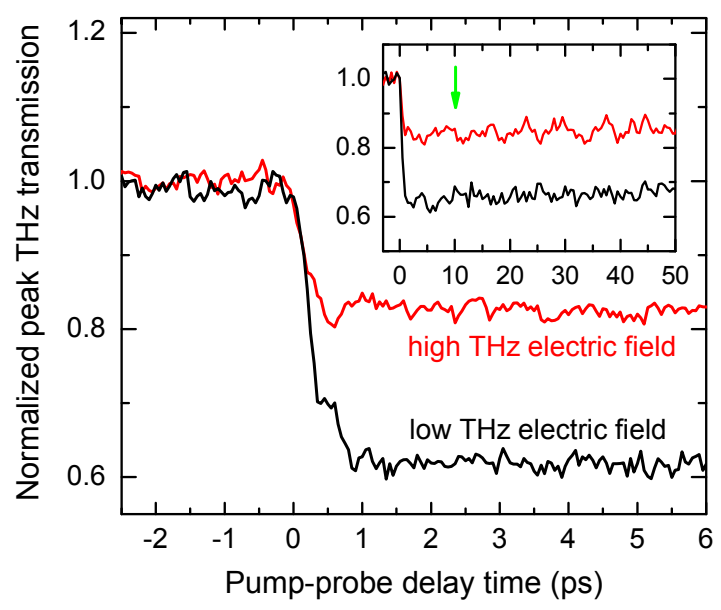

Figure 5. Normalized transmission of the peak electric field of a $\mathrm{THz}$ pulse at low and high $\mathrm{THz}$ fields as a function of delay time with respect to a $800 \mathrm{~nm}, 40 \mathrm{fs}$, optical pump pulse. The inset shows the corresponding long-term dynamics.

The $800 \mathrm{~nm}$ pump pulse photoexcites electron-hole pairs in the normally insulating GaAs sample. Since holes in GaAs have a significantly lower mobility when compared to electrons, we assume the contribution of the holes to the overall conductivity of the sample to be negligible. Photoexcited electrons are then injected into the higher mobility central Tvalley in the conduction band. At low THz probe fields, the transmission of the THz pulse through the photoexcited GaAs sample is approximately $60 \%$ of the transmission through the unexcited sample. However at high $\mathrm{THz}$ probe fields, the relative change in transmission is greatly reduced in comparison to the low $\mathrm{THz}$ probe field, suggesting that $\mathrm{THz}$ absorption bleaching is again taking place. As in the case of doped InGaAs, we suppose that the absorption 
bleaching observed at high THz probe fields in photoexcited GaAs is due to THz-pulse-induced intervalley scattering of electrons between the $\Gamma$. and L valleys of the conduction band. It is well-known that optically induced intervalley scattering in ultrafast pump-probe and OPTP experiments (performed using higher pump photon energies, e.g., $400 \mathrm{~nm}$ pump pulses) can excite carriers directly into the satellite valleys via optical phonon scattering [16,17]. In addition, the observation of electric-field-induced intervalley scattering has recently been reported in OPTP experiments in DC-biased GaAs wafers [18]. However, here we show intervalley scattering induced by the THz probe pulse itself in an OPTP experiment.

The mathematical model developed for the $\mathrm{Z}$ scan experiment [12], based on the Drude model and incorporating intervalley scattering, has been used to describe the photoexcitation experiment as well [15]. The contribution of the holes to the $\mathrm{THz}$ transmission mechanism has been found to be negligible, as expected, while a significant reduction of the intravalley scattering time at high $\mathrm{THz}$ fields (from $160 \mathrm{fs}$ down to 50fs) needs to be included to better fit the experimental data.

\section{CONCLUSIONS}

We have performed several nonlinear experiments on free-carriers in direct bandgap semiconductors at $\mathrm{THz}$ frequencies. Techniques as Z-Scan, THz-pump / THz-probe, and optical-pump / THz-probe have been employed to explore nonlinear interactions in both $\mathrm{n}$-doped and photoexcited samples. The mechanism that dominates this kind of interactions is found to be intervalley scattering and this effect, combined with a standard Drude-like response of free carriers in semiconductors, well explains the experimental results.

\section{REFERENCES}

[1] S. Mukamel, Principles of nonlinear spectroscopy, Oxford University Press, (1999).

[2] F. Blanchard et al., Opt. Exp., vol. 15, 13212 (2007).

[3] K.-L. Yeh, Appl. Phys. Lett., vol. 90, 171121 (2007).

[4] A. Mayer and F. Keilmann, Phys. Rev. B, vol. 33, 6954 (1986).

[5] A. Mayer and F. Keilmann, Phys. Rev. B, vol. 33, 6962 (1986).

[6] J. Hebling et al., IEEE J. Sel. Top. Quant. Electron., vol. 14, 345 (2008).

[7] Q. Wu and X. C. Zhang, Appl. Phys. Lett., vol. 68, 1604 (1996).

[8] M. Sheik-Bahae et al., IEEE J. Quantum Electron., vol. 26, 760 (1990).

[9] B. Gu et al., Appl. Phys. Lett., vol. 92, 091118 (2008).

[10] A. D. Lad et al., Appl. Phys. Lett., vol. 90, 133113 (2007).

[11] S. E. Ralph et al., Phys. Rev. B, vol. 54, 5568 (1996).

[12] L. Razzari et al., Phys. Rev. B, vol. 79, 193204 (2009).

[13] F. Blanchard et al., in preparation.

[14] M. Lundstrom, Fundamentals of carrier transport, Cambridge University Press, 2nd ed. Cambridge, U.K (1990).

[15] F. H. Su et al., Opt. Exp., vol. 17, 9620 (2009).

[16] D. G. Cooke et al., Appl. Phys. Lett., vol. 89, 122103 (2006).

[17] M. C. Nuss et al., Phys. Rev. Lett., vol. 58, 2355 (1987).

[18] Q.-L. Zhou et al., Appl. Phys. Lett., vol. 93, 102103 (2008). 